\title{
Definition of water droplets "strain cycles" in air times dependences on their sizes and movement velocities
}

\author{
Roman Volkov, Alena Zhdanova, Maxim Zabelin, Geniy Kuznetsov, and Pavel Strizhak ${ }^{\mathrm{a}}$ \\ National Research Tomsk Polytechnic University, 30, Lenin Avenue, 634050 Tomsk, Russia
}

\begin{abstract}
Experimental investigation of water droplets deformation regularities during their motion in the air by the action of gravitational forces was executed. Characteristic sizes of droplets were varied in the range from $3 \mathrm{~mm}$ to $6 \mathrm{~mm}$. Velocities of droplets movement attained to $5 \mathrm{~m} / \mathrm{s}$. The cross-correlation system of video registration was used. More than ten characteristic "strain cycles" of droplets during the $1 \mathrm{~m}$ distance motion by them thought the air were established. Characteristic of droplets forms, periods, dimensions and ranges were determined for all "strain cycles". "Strain cycle" times dependences on velocity and sizes of droplets were established.
\end{abstract}

\section{Introduction}

Experimental and theoretical investigations of particle (droplets) movement in gas flows (in particular, [1-3]) executed in the XIX-XX centuries allowed establishing the dependences of particle sizes, forms and properties and also gases properties on the main movement characteristics and modes. It is shown in [4] that the movement velocity of such particles (droplets) depends up a wide range of factors and is changed nonlinearly during the movement (more intensively under realization of phase transformations and chemical reaction).

Results of experimental investigations of the main characteristics of liquid droplets deformation (water with various inclusions and admixtures) under free falling in the air are adduced in [5-7]. The nature of the main liquid properties (viscosity, surface-tension, density) and droplet sizes influence on modifier condition of their form was determined. The substantiation attempt of expressions for deformation characteristics according to investigated factors [5-7] was made. The influence of movement velocity on a droplet form was not established. According to conclusions [1-3] it can be made the assumption about sufficiently significant influence of this parameter on deformation characteristics. Complex study of liquid droplet sizes and movement velocities influence on the modifier conditions of their configuration during flying through gas area is of interest.

It was established in [5-7] that droplets change their form during motion through the gas area cyclically. At that only the first "strain cycle" after the separation from the dosing device was investigated rather fully. It is expediently to conduct the investigations for several consequent "strain cycles" because the droplet velocities are changed nonlinear [5-7] during motion.

\footnotetext{
${ }^{\text {a }}$ Corresponding author: pavelspa@tpu.ru
}

This is an Open Access article distributed under the terms of the Creative Commons Attribution License 4.0, which permits unrestricted use, distribution, and reproduction in any medium, provided the original work is properly cited. 


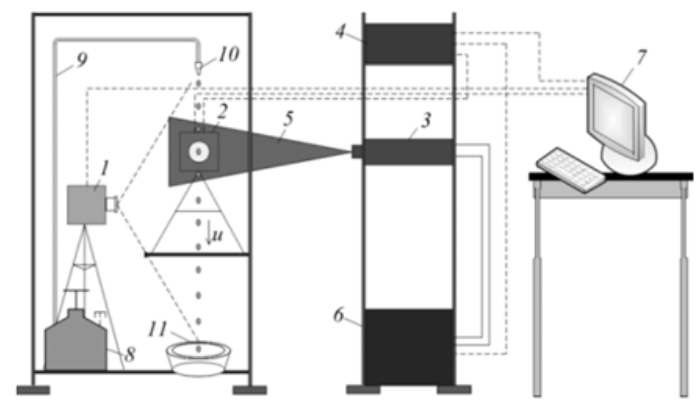

Figure 1. A scheme of experimental setup: 1 - high-velocity digital camera, 2 - cross-correlation digital camera, 3 - double pulsed solid-state laser, 4 - synchronizer of personal computer (PC), cross-correlation digital camera and laser, 5 - light "pulse", 6 - laser generator, 7 - PC, 8 - vessel with experimental liquid, 9 - channel of experimental liquid supply, 10 - dosing device, 11 - catcher.

At that the variation range of droplet velocities as well as their initial sizes should be constrained by the moderate condition of Weber's numbers (We $<7$ ). It is due to that fact that the probability of droplet breakage is high as consistent with the main lays and conclusions $[7,8]$ at $\mathrm{We}<7$.

The purpose of the work is experimental definition of approximating expressions for the main characteristics of water droplets "strain cycles" dependences on sizes and movement velocities of droplets.

\section{Experimental setup and methods}

The experimental setup (Fig. 1) was used. It provides the video registration of single water droplets motion of the $1 \mathrm{~m}$ distance from dosing device 10 to catcher 11 .

Initial water droplet sizes were varied in the range of $3 \div 6 \mathrm{~mm}$. The diameter $\left(d_{0}\right)$ of droplet at a separation from the dosing device 10 was accepted as its initial characteristic size. Experiments showed that the droplet has the form approximate to spherical at this timepoint. Initial droplet velocities $u_{0}$ were varied within the range of $0 \div 3 \mathrm{~m} / \mathrm{s}$. Increase of droplets velocities $u$ to $5 \mathrm{~m} / \mathrm{s}$ was provided at ejection of droplets with these velocities by the dosing device 10 on $1 \mathrm{~m}$ distance interval.

Maximum Weber's numbers in experiments were $\mathrm{We}_{\max }=u^{2} d_{0} \rho_{g} / \sigma_{l}=5^{2} \cdot 6 \cdot 10^{-3} \cdot 1.5 / 0.0618 \approx 3.64$ under gas (air) density $\rho_{a} \approx 1.5 \mathrm{~kg} / \mathrm{m}^{3}$ and liquid (water) surface tension coefficient $\sigma_{l}=0.0618 \mathrm{~kg} / \mathrm{s}^{2}$. It can be concluded on providing of the moderate Weber's numbers $(\mathrm{We}<7$ ) condition at which the probability of droplets breakage is extremely small $[7,8]$ in conducted experiments.

The following list was used (Fig. 1) as the registration equipment: digital video camera 1 with figure format of $1024 \times 1024$ pixels, frame frequency up to $10^{5}$ per second; cross-correlation digital camera 2 with figure format of $2048 \times 2048$ pixels, minimal delay between two sequence frames not more than $5 \mu$ s; double pulsed solid-state laser 3 with wave-length $532 \mathrm{~nm}$, energy in impulse not less than $70 \mathrm{~mJ}$, impulse time not more than $12 \mathrm{~ns}$, recurrence frequency not more than $15 \mathrm{~Hz}$; synchronizing processor 4 with signal sampling not more than $10 \mathrm{~ns}$.

The water droplets emanated from the dosing device 10 and flew the distance of $1 \mathrm{~m}$ in air to the catcher 11 according to the constrained initial sizes $d_{0}$ and velocity $u_{0}$. The process of droplets movement was registered by the digital camera $l$ with frame frequency up to $10^{4}$ per second. Not less than 10 experiments for one size and velocity of droplets under other fixed conditions were conducted. Sections with characteristic changes of droplet configuration were distinguished on the personal computer (PC) 7 after videogams processing. The time intervals $t_{\mathrm{d}}$ were fixed during which the droplets finish total "strain cycle" in other words they consistently twice take the form close to the 


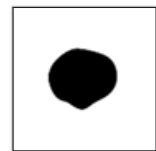

(a)

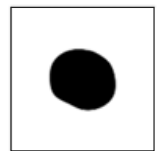

(b)

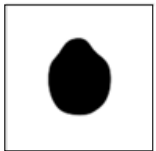

(c)

Figure 2. Typical water droplet forms in the first "strain cycle" at $d_{0}=3.5 \mathrm{~mm}$ and velocity at the inlet into the cycle $u=0.25 \mathrm{~m} / \mathrm{s}\left(t_{\mathrm{d}}=28.71 \mathrm{~ms}\right): \mathrm{a}-t=1.54 \mathrm{~ms}, \mathrm{~b}-t=8.02 \mathrm{~ms}, \mathrm{c}-t=18.39 \mathrm{~ms}$.

identical one. The distance between the dosing device 10 and catcher 11 was detached on the group of sections characterizing the corresponding "strain cycles" (their continuances $t_{\mathrm{d}}$ and extents $l_{\mathrm{d}}$ were calculated). The droplet size measurement was conducted for each marked section with use of crosscorrelation digital camera 2, laser 3, synchronizer 4. Ten experiments with identical sizes and velocities of droplets were conducted. Each videogram was divided on the estimated domains with dimensions of $32 \times 32$ pixels. The scaling coefficient $\gamma$ was calculated in consistent with recommendations [9]. The values of $\gamma$ were changed in the range of $0.01 \div 0.1 \mathrm{~mm} /$ pixel for videograms of the executed experiments. The nominal (as the droplets form in flight corresponds, generally, to ellipsoids) maximum droplet diameters in pixels were calculated with using of algorithms [9] and techniques [10,11] and then the recalculation in millimeters was carried out with coefficient $\gamma$ application. $d_{\mathrm{x}}$ - maximum transverse (relative to the movement direction) size of droplet, $\mathrm{mm}, d_{\mathrm{y}}$ - maximum longitudinal size of droplet, $\mathrm{mm}, d_{\max }$-absolute maximum size of droplet, $\mathrm{mm}$ were calculated. Accuracy errors of the maximum characteristic droplet sizes measurement were $10^{-5} \mathrm{~m}$, they were determined by techniques [9-11]. The relative variables: $\Delta_{x}=\left(d_{\mathrm{x}}-d_{0}\right) / d_{0}, \Delta_{\mathrm{y}}=\left(d_{\mathrm{y}}-d_{0}\right) / d_{0}, \Delta_{\max }=\left(d_{\max }-d_{0}\right) / d_{0}$ were used for an estimation of droplet sizes change during deformation.

The $t_{\mathrm{d}}$ and $l_{\mathrm{d}}$ values received at all distance covered by a droplet registration by the video camera were specified for each "strain cycle" at the determined $d_{\mathrm{x}}, d_{\mathrm{y}}$ and $d_{\max }$ parameters by results of crosscorrelation camera 2 videogram processing. The accuracy errors of time $t_{\mathrm{d}}$ and extents $l_{\mathrm{d}}$ measurement by the cross-correlation digital camera at $\gamma=0.01 \div 0.1 \mathrm{~mm} /$ pixel did not exceed $10^{-5} \mathrm{~s}$ and $10^{-4} \mathrm{~m}$ appropriately.

\section{Results and discussion}

It was established at processing of executed experiment videograms that water droplets during motion (at $d_{0}=3 \div 6 \mathrm{~mm}$ and $u=0 \div 5 \mathrm{~m} / \mathrm{s}$ ) from the dosing device 10 to catcher 11 (Fig. 1 ) carry out more than ten "strain cycles" with characteristic times $t_{\mathrm{d}} \ll 1 \mathrm{~s}$ and extents $l_{\mathrm{d}}$ of each from $10 \mathrm{~mm}$ to $190 \mathrm{~mm}$. Thus it was revealed that droplets removal from the dosing device 10 is characterized by nonlinear increase of $l_{d}$ values and decrease of $t_{\mathrm{d}}$. It was also established that the number of specific droplet forms decreases for purposes of every consequent "strain cycle".

Some droplet forms which are typical for the first (since the separation from the dosing device 10) "strain cycle" at $d_{0}=3.5 \mathrm{~mm}$ and $u_{0}=0 \mathrm{~m} / \mathrm{s}$ (correspond to conditions of their free falling in air) are presented in Fig. 2.

It can be drawn the conclusion about good correlation of the received videograms with experiment [5] results of regularities study of droplet free falling with water-glyceric (concentration of glycerin was changed in the range of $0 \div 91 \%$ ) compositions in air. Six characteristic forms of water droplets with sizes $d_{0} \approx 5.1 \mathrm{~mm}$ were adduced in [5] for the first "strain cycle". Similar six droplet forms were established in conducted experiments (three of them are adduced in Fig. 2). Thus delay times between these forms (Fig. 2) are in good agreement with [5]. Rangeability values of droplet sizes and extent of concerned "strain cycle" aren't assumed in [5] therefore the comparison to times $t_{\mathrm{d}}$ and characteristic 


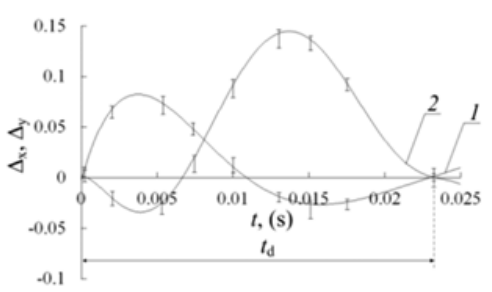

(a)

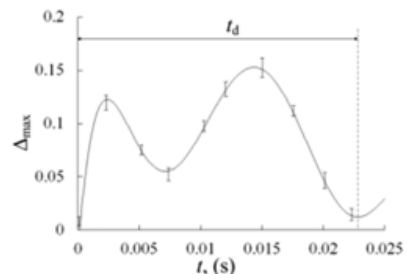

(b)

Figure 3. Change of strain range within limits of third "strain cycle" at $d_{0}=3.5 \mathrm{~mm}$ and velocity at the inlet into cycle $u=1 \mathrm{~m} / \mathrm{s}: 1-\Delta_{\mathrm{x}}, 2-\Delta_{y}$.

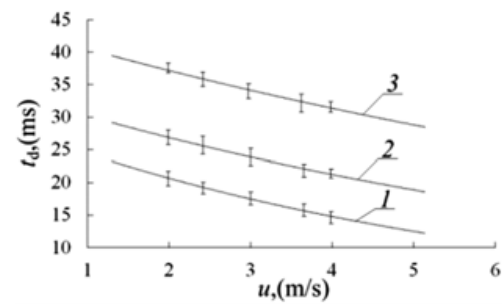

(a)

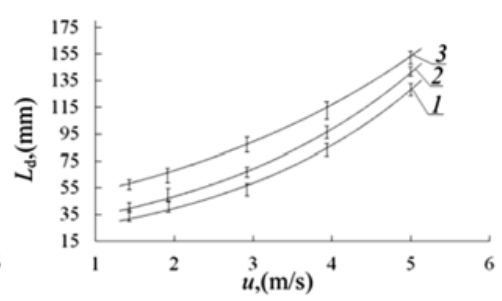

(b)

Figure 4. Dependences of $t_{\mathrm{d}}$ (a) and $l_{\mathrm{d}}$ (b) on velocities $u$ at the inlet into "strain cycles" at $d_{0}=3 \mathrm{~mm}(1)$, $d_{0}=4 \mathrm{~mm}(2)$ and $d_{0}=5 \mathrm{~mm}(3)$.

droplet forms is possible only. Values of $t_{\mathrm{d}}$ for executed experiments with $d_{0}=5 \mathrm{~mm}$ and $u_{0}=0 \mathrm{~m} / \mathrm{s}$ were about $36 \mathrm{~ms}$. Value (38 $\mathrm{ms})$ is adduced in [5].

Experiments showed that $t_{\mathrm{d}}$ times decrease, and values of rangeabilities $\left(\Delta_{\mathrm{x}}, \Delta_{\mathrm{y}}, \Delta_{\max }\right)$ of droplets sizes increase with every consequent "strain cycle". Specifically, this is due to increase of droplet movement velocities and effect of movement resistant force. Typical changes of $\Delta_{\mathrm{x}}, \Delta_{\mathrm{y}}, \Delta_{\max }$ at $d_{0}=3.5 \mathrm{~mm}$ and velocity $u=1 \mathrm{~m} / \mathrm{s}$ at the inlet into "strain cycle" are adduced in Fig. 3 .

The range $\Delta_{\max }$ attained the maximum values 0.21 at change of the droplets sizes and velocities in the intervals of $3 \div 6 \mathrm{~mm}$ and $u=0 \div 5 \mathrm{~m} / \mathrm{s}$. Minimum and maximum values of $t_{\mathrm{d}}$ were $16 \mathrm{~ms}$ and $42 \mathrm{~ms}$, respectively. Values $12 \mathrm{~mm}$ and $184 \mathrm{~mm}$ can be noted as minimum and maximum ones for $l_{\mathrm{d}}$.

Dependences of $t_{\mathrm{d}}$ and $l_{\mathrm{d}}$ on velocities of droplets movement $u$ for various $d_{0}$ values are adduced in Fig. 4.

Analysis of Fig. 4 allows drawing the inference about significant influence on deformation characteristics not only $u$ velocities, but also the initial $d_{0}$ sizes. In particular, it was established that $\Delta_{\max }$ values attains 0.14 at $d_{0}=3 \mathrm{~mm}(u=1 \mathrm{~m} / \mathrm{s})$, and this parameter not to exceed 0.2 at $d_{0}=6 \mathrm{~mm}$. The reason for this is distinctly that the mass and deformation persistence of droplets increase with rising of its sizes.

Experiment results of $t_{\mathrm{d}}$ establishment at $u=0 \div 5 \mathrm{~m} / \mathrm{s}$ and $d_{0}$ variability in the range from $3 \mathrm{~mm}$ to $6 \mathrm{~mm}$ (in comparison to findings [5]) are provided in Fig. 5.

Comparison of curves 1 and 2 shows that $t_{\mathrm{d}}$ times are in sufficiently good correlation with experimental findings [5]. At the same time it should be noted that $t_{\mathrm{d}}$ times [5] exceed that one which received in executed experiments under identical conditions $\left(u_{0} \rightarrow 0 \mathrm{~m} / \mathrm{s}\right)$. The analysis of the video frames at the time of droplet separation from the dosing device showed that some time interval which includes directly the separation process comes before the droplet form taken as initial one in [5]. This time interval increases from $5 \mathrm{~ms}$ to $12 \mathrm{~ms}$ with rising of the droplets sizes. The values of $t_{\mathrm{d}}$ received in 


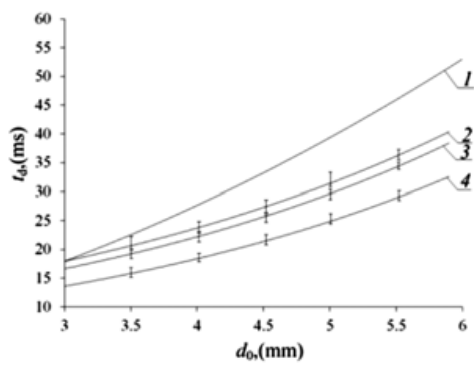

Figure 5. Times $t_{\mathrm{d}}$ at various water droplets sizes: 1 - approximating curve [5]; 2-4 - approximations of experiments results when velocities at the inlet into "strain cycle" $u=1 \mathrm{~m} / \mathrm{s}, u=3 \mathrm{~m} / \mathrm{s}$ and $u=5 \mathrm{~m} / \mathrm{s}$, respectively.

concerned experiments and [5] will be different less than by $3 \%$ if these times will be included in the first "strain cycle".

It is also possible to note that velocities of droplets at the inlet into the first "strain cycle" are different from experiments [5]. It was established from analysis of videograms [5] that these differences attain $6 \%$. Probably $t_{\mathrm{d}}$ values in conducted experiments are different (Fig. 5) from results [5] for these two reasons.

Comparison of $t_{\mathrm{d}}=f\left(d_{0}\right)$ curves at various $u$ (Fig. 5) shows that increase of droplets movement velocities leads to quite considerable decrease of characteristic times $t_{\mathrm{d}}$.

The established features of droplet movement velocities influence on $t_{\mathrm{d}}$ times allow carrying the inference about significant restrictions on use of expressions presented in [5], for calculation of first "strain cycles" times as "periods of natural oscillations":

$$
t_{\mathrm{d}}=\frac{\pi}{4} \frac{\rho_{1} \delta^{2}}{\eta_{1}} \frac{1}{\sqrt{\mathrm{Lp}-6.25}},
$$

where $\rho_{l}$ - liquid density, $\mathrm{kg} / \mathrm{m}^{3} ; \delta$ - characteristic droplet size, $\mathrm{mm} ; \eta_{l}$ - dynamic viscosity of liquid, $\mathrm{kg} /(\mathrm{m} \cdot \mathrm{s}) ; \mathrm{Lp}$ - Laplace's number $\left(\mathrm{Lp}=\delta \cdot \rho_{l} \cdot \sigma_{l} / \eta_{l}^{2}\right)$.

It is visible that $t_{\mathrm{d}}$ time represents the function of liquid sizes and properties (viscosity, a superficial tension, density). A velocity of droplets movement isn't considered.

It was established in executed experiments that the droplet movement velocity can be changed sufficiently significantly (to 30\%) even within limits of one "strain cycle" and by several times when passing more than ten such cycles. This effect essentially influences on characteristics of "strain cycles" (Fig. 5) at $t_{\mathrm{d}} \ll 1 \mathrm{~s}$.

Approximating expressions for $t_{\mathrm{d}}$ times depending on the velocity droplets at the inlet into "strain cycle" and also the initial sizes were received when processing of experiment results.

For example:

$$
\begin{gathered}
t_{\mathrm{d}}=0.0257-0.0022 u \text { at } d_{0}=3 \mathrm{~mm}, 0<u<5 \mathrm{~m} / \mathrm{s} \\
t_{\mathrm{d}}=0.0453-0.0018 u \text { at } d_{0}=6 \mathrm{~mm}, 0<u<5 \mathrm{~m} / \mathrm{s} \\
t_{\mathrm{d}}=0.0027 d_{0}^{2}-0.0174 d_{0}+0.0535 \text { at } u=1 \mathrm{~m} / \mathrm{s}, 3<d_{0}<6 \mathrm{~mm} ; \\
t_{d}=0.0022 d_{0}^{2}-0.012 d_{0}+0.0302 \text { at } u=5 \mathrm{~m} / \mathrm{s}, 3<d_{0}<6 \mathrm{~mm} .
\end{gathered}
$$

It is important to note that approximations (2)-(5) are presented for $t_{\mathrm{d}}$ times depending on velocity at the inlet into "strain cycles" and the initial size $d_{0}$. Velocity of droplets during the movement process 


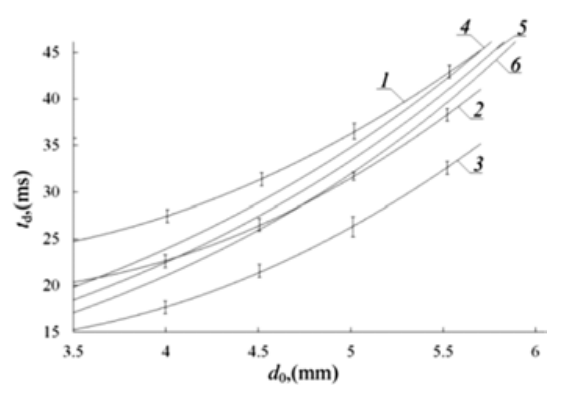

Figure 6. Times $\left(t_{d}\right)$ at various sizes and velocities of water droplets: $1-3-$ approximations of experiment results when velocities at the inlet into "strain cycle" $u=1 \mathrm{~m} / \mathrm{s}, u=3 \mathrm{~m} / \mathrm{s}$ and $u=5 \mathrm{~m} / \mathrm{s}$, respectively, 4-6-times values $t_{\mathrm{d}}$ calculated with use of expression (6).

increases nonlinear. Therefore it is quite difficult to assume the $t_{\mathrm{d}}$, as function of $u=f(t)$. The account of $d_{\mathrm{x}}=f(t), d_{\mathrm{y}}=f(t)$ and $d_{\max }=f(t)$ dependences is of even great difficulty as a consequence of continuous deformation.

It should be noted the important feature of the theoretical conclusions [5]. Expression (1) was formulated for droplets in the form of the sphere (there is a multiplier $\pi \delta^{2} / 4$ ). Experiments executed within this work showed that the droplet has a spherical form not more often than the form of ellipsoid, "plate", elongate cylinder and other rotation bodies (Fig. 2) in the process of deformation. Therefore it is expediently to calculate the $S_{\mathrm{d}}$ droplet area at the beginning of every "strain cycle" (and/or consider its change in the cycle $S_{\mathrm{d}}=f(t)$ ) for maximum possible approximation of expression (1) to real values of $t_{\mathrm{d}}$.

The analysis of approximating expressions (2)-(5) allows drawing the conclusion that $t_{\mathrm{d}}$ values may be calculated with use of expression (1) at $u \rightarrow 0 \mathrm{~m} / \mathrm{s}$. The first summands in formulas (2)-(5) are practically (deviations up to 7\%) equal to the $t_{\mathrm{d}}$ values determined with use of expression (1) for corresponding characteristic droplets sizes $d_{0}$. The marked deviations may be reduced to $3 \div 4 \%$ when using the values of the droplet areas at the inlet into "strain cycle" $\left(S_{\mathrm{d}}\right)$ determined according to real droplet forms instead of a multiplier $\pi \delta^{2} / 4$ in expression (1).

Dependences $t_{\mathrm{d}}=f(u)$ represent polynomial functions of the first order (2)-(5) as a first approximation. Thus the analysis of multipliers before $u$ velocity in expressions (2)-(5) shows that they depend on the droplets sizes rather significantly.

The second summands in expressions (2)-(5) can be classified rather definitely by the expression $\sqrt{\frac{\mathrm{We}}{\mathrm{Lp}-6.25}}$. It was established as a result of iterative determines at $d_{0}=3 \div 6 \mathrm{~mm}$ and $u=$ $0 \div 5 \mathrm{~m} / \mathrm{s}$. In such a way, the formula for calculation of "strain cycle" times with consideration for droplets form, their sizes, movement velocities, properties of liquid and gas is as follows:

$$
t_{\mathrm{d}}=\frac{\rho_{1} S_{d}}{\eta_{1} \sqrt{\mathrm{Lp}-6.25}}-\sqrt{\frac{\mathrm{We}}{\mathrm{Lp}-6.25}} .
$$

The $t_{\mathrm{d}}$ values received with use of expression (6) at $d_{0}=3 \div 6 \mathrm{~mm}, u=1 \div 5 \mathrm{~m} / \mathrm{s}, \rho_{l}=1000 \mathrm{~kg} / \mathrm{m}^{3}$, $\sigma_{l}=0.0618 \mathrm{~kg} / \mathrm{m}^{2}, \eta_{l}=0.001023 \mathrm{~kg} /(\mathrm{m} \cdot \mathrm{s}), \rho_{g}=1.5 \mathrm{~kg} / \mathrm{m}^{3}$ and $S_{\mathrm{d}}=\pi d_{0}^{2} / 4$, are presented in Figure 6 for comparison with experimental approximating dependences.

Figure 6 illustrates that expression (6) can be used as the first approximation to establish the water droplet "strain cycles" times under concerned conditions. Deviations of the determined values $t_{\mathrm{d}}$ from the experimental ones do not exceed $6 \%$ at average movement velocities $(2 \div 4 \mathrm{~m} / \mathrm{s})$. These deviations can be reputed reasonable if the presented confidence ranges (illustrate the errors of measurements in experiments) will be consider. Deviations from experimental values $t_{\mathrm{d}}$ are sensibly significant at "low" 
(about $1 \mathrm{~m} / \mathrm{s}$ ) and "high" (about $5 \mathrm{~m} / \mathrm{s}$ ) velocities at the inlet into "strain cycles". This result allows drawing the inference about expression (6) frontiers up on velocities of droplets movement.

\section{Conclusions}

Known [5] expressions for the main characteristic of "strain cycle" $-t_{\mathrm{d}}$ time can be used only at "less" ( $u \ll 1 \mathrm{~m} / \mathrm{s}$ ) velocities of droplets movement. Expression (6) can be used, as a first approximation for the predictive analysis of $t_{\mathrm{d}}$ times at velocities of water droplets movement in the air, changing in the range of $0-5 \mathrm{~m} / \mathrm{s}$.

This work was financially supported by the Russian Foundation for Basic Research (project No. 14-08-00057).

\section{References}

[1] A.B. Basset, Phil. Trans. Roy. Soc. A179 (1888)

[2] C.W. Oseen, Hydromechanik (Akademische Verlagsgem, Leipzig, 1927)

[3] W.A. Sirignano, Fluid Dynamics and Transport of Droplets and Sprays (Cambridge University, 1999)

[4] V.I. Terekhov, M.A. Pakhomov, Heat and mass transfer and hydrodynamics in gas-steam flows (Novosibirsk, Novosibirsk State Technical University, 2009) (in Russian)

[5] V.V. Dubrovskii, A.M. Podvysotskii, A.A. Shraiber, J. Eng. Phys. 58, 5 (1990)

[6] A. Wierzba, Exp. in Fluids. 9, 1 (1990)

[7] A.A. Shreiber, A.M. Podvisotski, V.V. Dubrovski, Atom. and Sprays. 6, 6 (1996)

[8] A.K. Flock, D.R. Guildenbecher, J. Chen, P.E. Sojka, H.J. Bauer, Int. J. Mult. Flow. 47 (2012)

[9] M. Raffel, C. Willert, J. Kompenhans, Particle image velocimetry (Berlin, Springer Verlag, 1998)

[10] R.S. Volkov, O.V. Vysokomornaya, G.V. Kuznetsov, P.A. Strizhak, J. Eng. Phys. and Therm. 86, $6(2013)$

[11] A.V. Zakharevich, P.A. Strizhak, J. Eng. Therm. 22, 2 (2013) 